\title{
SELF MAPS OF PROJECTIVE SPACES
}

\author{
BY \\ C. A. MCGIBBON ${ }^{1}$
}

\begin{abstract}
The classical projective $n$-spaces (real, complex, and quaternionic) are studied in terms of their self maps, from a homotopy point of view. Self maps of iterated suspensions of these spaces are also considered. The goal in both cases is to classify, up to homology, all such maps. This goal is achieved in the stable case. Some partial results are obtained in the unstable case. The results from both cases are used to compute the genus groups and the stable genus groups of the classical projective spaces. Applications to other spaces are also given.
\end{abstract}

1. Summary. Let $P^{n}$ denote a projective $n$-space (either real, complex, or quaternionic). This paper deals with the classification, up to homology, of self maps

$$
f: P^{n} \rightarrow P^{n} \text { and } g: \Sigma^{k} P^{n} \rightarrow \Sigma^{k} P^{n} .
$$

Here $\Sigma^{k} P^{n}$ denotes the $k$-fold suspension of $P^{n}$. We shall assume that $k$ is large with respect to $n$ in order to make the second classification a stable one.

It is clear that these two problems are related. Indeed for any space $X$ and self maps $f_{i}, i=1, \ldots, m$, one can use the track addition of maps on $\Sigma^{k} X$ to form a linear combination of $k$-fold suspensions

$$
n_{1} \Sigma^{k} f_{1}+\cdots+n_{m} \Sigma^{k} f_{m}: \Sigma^{k} X \rightarrow \Sigma^{k} X
$$

Under what conditions can every self map of $\Sigma^{k} X$ be described, up to homology, in this manner? The following result answers this question for the classical projective spaces. In its statement, two maps are called homologous if they induce the same homomorphism in integral homology.

THEOREM 1. If $k$ is large with respect to $n$, then every self map of $\Sigma^{k} P^{n}$ is homologous to a linear combination of $k$-fold suspensions, if and only if

(i) $P^{n}=\mathbf{R} P^{n}$ and $n \neq 3$ or 7 , or

(ii) $P^{n}=\mathbf{C} P^{n}$ for all $n$, or

(iii) $P^{n}=\mathbf{H} P^{n}$ and $n<4$.

This theorem is a corollary of some of the results that we obtain in the next two sections. In $\S 2$, the unstable classification problem is considered. There the quaternionic case receives special attention. Unfortunately, the classification of self maps

Received by the editors February 18, 1981 and, in revised form, May 22, 1981.

1980 Mathematics Subject Classification. Primary 55S37; Secondary 55R35, 55P42, 55N15.

$K e y$ words and phrases. Projective $n$-space, suspension, Adams $e$-invariant, Hopf bundle, Vandermonde determinant, Maclaurin's series, genus of a nilpotent homotopy type, Lefschetz number, order of the identity.

${ }^{1}$ Supported in part by NSF. 
of $\mathbf{H} P^{n}$ is still an open problem. Feder and Gitler [11] have conjectured a classification in terms of certain congruences. We describe their conjecture and prove some special cases of it.

In $\$ 3$ we solve the stable classification problem for all three families of projective spaces. In other words, we classify those endomorphisms of $\tilde{H}_{*}\left(P^{n} ; \mathbf{Z}\right)$ that are induced by maps $\Sigma^{k} P^{n} \rightarrow \Sigma^{k} P^{n}$, where $k$ is sufficiently large. In doing so we treat the complex and quaternionic cases simultaneously. These two cases have more in common than Theorem 1 would suggest. In both cases such an endomorphism corresponds to a sequence of $n$ integers. The set of all sequences so induced forms a subgroup of finite index in $\mathbf{Z}^{n}$. We describe this subgroup in some detail. The key tool in our description is Theorem 3.6, which describes a group isomorphism between this subgroup and the appropriate $K$-theory of $P^{n}$.

$\S 4$ is devoted to applications. The first application is to classify those integers $n$ for which every stable self map of $P^{n}$ has a nonzero Lefschetz number (and hence a fixed point).

We then recall the notion of the genus of a nilpotent homotopy type. We compute genus group $G\left(P^{n}\right)$ and stable genus group $G S\left(P^{n}\right)$ for all cases in which these groups can be defined.

We also consider the Lie group $S U(n)$. Zabrodsky has given a lower bound on the order of $G(S U(n))$ [32, p. 152], which appears to be sharp. We use our results on $G S\left(\mathbf{C} P^{n-1}\right)$ to give a new proof of his estimate.

The last application concerns the order of the identity map in the iterated loop space of the connective cover $\Omega^{k} S^{2 n-1}\langle 2 n-1\rangle$, localized at a single prime $p$. We use our results on self maps of $\Sigma^{m} \mathbf{C} P^{\infty}$, to prove a theorem of Neisendorfer and Selick [21]. This theorem states that for the loop space in question, the identity map does not have finite order if $k<2 n-3$.

I would like to thank J. Eric Brosius, Chuck Weibel, and Joe Neisendorfer for their help with various parts of this paper.

2. The unstable problem. Most of the results in this section deal with the classification of self maps of quaternionic projective $n$-space. However, let us first review what is known in the real and complex cases.

THEOREM 2.1. For each integer $n \geqslant 1$,

$$
\left[\mathbf{R} P^{n}, \mathbf{R} P^{n}\right] \simeq \begin{cases}\mathbf{Z} & \text { if } n \text { is odd } \\ \mathbf{Z} / 2 & \text { if } n \text { is even }\end{cases}
$$

This classification is faithfully represented in homology; for each $\lambda$ in $\mathbf{Z}$ (if $n$ is odd) or in $\mathbf{Z} / 2$ (if $n$ is even) there is a self map $f$ such that $f_{*} x=\lambda x$ for all $x \in \tilde{H}_{*}\left(\mathbf{R} P^{n} ; \mathbf{Z}\right)$.

TheOREM 2.2. For all $n \geqslant 1,\left[\mathbf{C} P^{n}, \mathbf{C} P^{n}\right] \simeq \mathbf{Z}$. Moreover, to each integer $\lambda$, there corresponds a self map which, for all $k$, induces multiplication by $\lambda^{k}$ on $H_{2 k}\left(\mathbf{C} P^{n} ; \mathbf{Z}\right)$. 
Both of these theorems can be proved using first principles in algebraic topology and so their proofs are omitted. We now turn to the quaternionic case where the complete answer is still unknown.

Since $H^{*} \mathbf{H} P^{n} \simeq \mathbf{Z}[u] / u^{n+1}$ where $u$ is a generator in dimension 4, a self map of $\mathbf{H} P^{n}$ is determined, up to homology, by its degree in this dimension. This prompts the following

Definition. An integer $\lambda$ will be called $n$-realizable if there is a self map of $\mathbf{H} P^{n}$ with degree $\lambda$ in dimension 4 .

The homology classification of self maps of $\mathbf{H} P^{n}$ thus amounts to a characterization of such integers. Using complex and quaternionic $K$-theory, Feder and Gitler [11] showed that an $n$-realizable integer must satisfy the following integrality conditions.

THEOREM 2.3. For $k \geqslant 1$, let $C_{k}$ denote the congruence

$$
\prod_{i=0}^{k-1}\left(x-i^{2}\right) \equiv 0 \quad \bmod \begin{cases}(2 k) ! / 2 & \text { if } k \text { is odd }, \\ (2 k) ! & \text { if } k \text { is even } .\end{cases}
$$

If $\lambda$ is $n$-realizable, then $\lambda$ is a solution to each of the congruences $C_{1}, C_{2}, \ldots, C_{n}$.

Feder and Gitler conjectured in [11] that these conditions are also sufficient (i.e., that any common solution to $C_{1}, C_{2}, \ldots, C_{n}$ is $n$-realizable). To date this conjecture has been verified only in the four cases; $n=1,2,3$ and $\infty[4,16,30]$. The last case is especially interesting. It was shown in [11] that if $\lambda$ is a solution to $C_{k}$ for all $k$, then $\lambda$ equals zero or an odd square. Sullivan had shown previously these integers are $\infty$-realizable.

There are two results in this paper which concern the Feder-Gitler conjecture. In the next section (Theorem 3.5) we show that each common solution $\lambda$ corresponds to a self map of $\Sigma^{k} \mathbf{H} P^{n}$ of the appropriate form. The following result may also be regarded as supporting evidence. Incidently, this next result gives us many examples of $n$-realizable integers which are not $\infty$-realizable.

Proposition 2.4. (i) Assume that $(\lambda, p)=1$ for each prime $p \leqslant 2 n$. Then the following three statements are equivalent:

(a) $\lambda$ is a solution to $C_{1}, C_{2}, \ldots, C_{n}$.

(b) $\lambda$ is $n$-realizable.

(c) $\lambda \equiv x^{2} \bmod p$ for each odd prime $p<2 n$ and $\lambda \equiv 1 \bmod 8$ if $n \geqslant 2$.

(ii) For each $n<\infty$ and even integer $\lambda$, some power of $\lambda$ is $n$-realizable.

If two self maps of $\mathbf{H} P^{n}$ induce the same homomorphism in homology does it follow that they are homotopic? Marcum and Randall [15] answered this question in the negative. For $n=3,4$ and 5 they found an essential self map of $\mathbf{H} P^{n}$ which induces the zero map in homology. There are good reasons to suspect that these maps are not isolated exceptions. Therefore, with regard to a homotopy classification of self maps of $\mathbf{H} P^{n}$ it appears that the next result is the best general result we are likely to get. 
THEOREM 2.5. For $n \leqslant \infty$, two self maps of $\mathbf{H} P^{n}$ induce the same homomorphism in homology if and only if they are stably homotopic.

Proof of Proposition 2.4. In (i), the implication (a) $\Rightarrow$ (c) follows from a glance at the congruences $C_{k}$. If $\lambda$ satisfies the conditions of (c) then by Rector [22], for each $p<2 n$ there is a self map of $\mathbf{H} P_{(p)}^{\infty}$ with degree $\lambda$ in dimension 4 . By restriction we thus obtain self maps of $\mathbf{H} P_{(p)}^{n}$ with degree $\lambda$ for all primes $<2 n$. At primes $p>2 n, \mathbf{H} P_{(p)}^{n}$ has the homotopy type of the $4 n$-skeleton of $K\left(\mathbf{Z}_{(p)}, 4\right)$ and so $\lambda$ is $n$-realizable at these primes also. These maps on the various localizations agree on the rational homotopy type. Therefore by the Pullback Theorem [12, p. 82] the implication (c) $\Rightarrow(b)$ is proved. The remaining step, (b) $\Rightarrow(a)$, is, of course, Theorem 2.3.

To prove (ii), assume that $f$ is a self map of $P^{n-1}=\mathbf{H} P^{n-1}$ with degree $\lambda$ in dimension 4. Let $\gamma$ represent the attaching map for the $4 n$-cell in $P^{n}$. Then $f$ extends to a self map of $P^{n}$ if and only $f_{\#} \gamma$ is a multiple of $\gamma$ in $\pi_{4 n-1} P^{n-1}$. We claim that $f_{\#}(\gamma)=\lambda^{n} \gamma+g$, where $g$ is a class with finite order. To see this, consider the long exact sequence of homotopy groups for the fibration $\gamma$. The fiber is $S^{3}$ and so $\pi_{4 n-1} P^{n-1} \approx \mathbf{Z} \oplus \pi_{4 n-2} S^{3}$. The first summand is generated by $\gamma$ and the second summand is a finite group. The coefficient $\lambda^{n}$ can be verified by rationalizing $P^{n-1}$. This process kills off the torsion and shows that $f_{0}$ extends to a self map of $P_{(0)}^{n}$. The coefficient in question then equals the degree of the extension in dimension $4 n$. By a cohomology calculation, this degree must be $\lambda^{n}$.

Since the class $g$ has finite order, there is a suspended class on $S^{4}$ whose image under the composition $\pi_{*} S^{4}=\pi_{*} P^{1} \rightarrow \pi_{*} P^{n-1}$ is $g$. In particular, this implies that $f_{\#}(g)=\lambda g$. If $f^{(k)}$ denotes the $k$-fold iterate of $f$, it then follows by induction on $k$ that

$$
f_{\#}^{(k)}(\gamma)=\lambda^{n k} \gamma+\lambda^{k-1} \sum_{i=0}^{k-1} \lambda^{i(n-1)} g
$$

A simple number theory exercise now shows that $k$ can be chosen so that the coefficient on $g$ is a multiple of the order of $g$. By the above remarks it follows that for this value of $k, \lambda^{k}$ is $n$-realizable.

Proof of Theorem 2.5. By the Cellular Approximation Theorem, it suffices to consider two maps $f, g: P^{n} \rightarrow P^{\infty}$ which agree in homology. Clearly $f$ and $g$ are stably homotopic iff $E^{\infty} f=E^{\infty} g$ in $\left[P^{n}, Q\left(P^{\infty}\right)\right]$. J. Becker showed in [6] that $B S p$ is a retract of $Q\left(P^{\infty}\right)$ and I showed in [17, Proposition 3.3] that the infinite suspension $E^{\infty}: P^{\infty} \rightarrow Q\left(P^{\infty}\right)$ factors through $B S p$. Since $K S p\left(P^{n}\right)$ is torsion free, the result follows.

3. The stable classification. We shall consider the real projective $n$-spaces first. The cases $n=3$ and 7 are exceptional because then $\mathbf{R} P^{n}$ is reducible after two suspensions. The proof of this for $n=3$ is standard. For $n=7$, this was proved by Rees [23], using the results of Milgram and Rees [18].

Notice that the following theorem is a bit sharper than Theorem 1(i). 
THEOREM 3.1. If $n \neq 3$ or 7 , then each self map of $\Sigma^{k} \mathbf{R} P^{n}$ is homologous to a $k$-fold suspension. However, when $n=3$ or 7 and $k>1$, the idempotents associated with the splitting $\Sigma^{k} \mathbf{R} P^{n} \simeq S^{k+n} \vee \Sigma^{k} \mathbf{R} P^{n-1}$ are not homologous to linear combinations of $k$-fold suspensions.

From a stable point of view, the complex and quaternionic projective spaces have many features in common. Our notation will attempt to reflect these similarities. Henceforth let $F=\mathbf{C}$ or $\mathbf{H}$ and let $d$ denote the dimension of $F$ over $\mathbf{R}$.

Let us first review some basics: The projective space $F P^{n-1}$ may be regarded as the orbit space of a free left action of the unit sphere in $F$ on $S^{d n-1}$. Let

$$
F \gamma_{n}: S^{d n-1} \rightarrow F P^{n-1}
$$

denote the quotient map of this action. It is well known that the map $F \gamma_{n}$ can be regarded as the attaching map for the $d n$-cell in a $\mathrm{CW}$ decomposition of $F P^{\infty}$.

The following numbers will play an important role in our calculations.

THEOREM 3.2. Let $\left|F \gamma_{n}\right|$ denote the order of the image of $F \gamma_{n}$ is the stable homotopy group $\pi_{d n-1}^{s} F P^{n-1}$. Then for $n \geqslant 1$

$$
\left|\mathbf{C} \gamma_{n}\right|=n !
$$

and

$$
\left|\mathbf{H} \gamma_{n}\right|= \begin{cases}(2 n) ! & \text { if } n \text { is even } \\ (2 n) ! / 2 & \text { if } n \text { is odd }\end{cases}
$$

For $n=2$, these orders are of course classical [31]. The other orders may be known to the experts but I have been unable to find them stated in the literature.

Given a self map $g$ of $\Sigma^{k} F P^{n}$, let $\delta(g)=\left(\delta_{1}(g), \ldots, \delta_{n}(g)\right)$ where $\delta_{i}(g)$ denotes the degree in homology of $g_{*}$ in dimension $k+d i$.

Definition. $D(n, F)=\left\{\delta(g) \mid g: \sum^{k} F P^{n} \hookleftarrow\right.$, and $\left.k>d n\right\}$.

The letter $D$ is meant to suggest degrees. Clearly $D(n, F)$ may be identified with the image of the homomorphism

$$
\lim _{\rightarrow}\left[\Sigma^{k} X, \Sigma^{k} X\right] \stackrel{f \rightarrow f}{\rightarrow}{ }^{*} \operatorname{Hom}\left(\tilde{H}_{*} X, \tilde{H}_{*} X\right)
$$

in the special case $X=F P^{n}$. Since $\operatorname{Hom}\left(\tilde{H}_{*} F P^{n}, \tilde{H}_{*} F P^{n}\right) \simeq \mathbf{Z}^{n}$, we may view $D(n, F)$ as a subgroup of $\mathbf{Z}^{n}$. Notice that the group structure on $D(n, F)$, which is induced by the stable track addition of maps, is compatible with the usual vector addition in $\mathbf{Z}^{n}$.

Here are some basic properties of the groups $D(n, F)$.

THEOREM 3.3. (i) For each $n \geqslant 1$, there is an epimorphism $D(n+1, F) \rightarrow D(n, F)$ which is induced by the restriction of self maps.

(ii) If $\left(0, \ldots, 0, \lambda_{m}, \ldots, \lambda_{n}\right) \in D(n, F)$, then $\left|F \gamma_{m}\right|$ divides $\lambda_{i}$ for $i \geqslant m$. Among such classes, the case $\lambda_{m}=\left|F \gamma_{m}\right|$ occurs in $D(n, F)$.

(iii) The index of $D(n, F)$ in $\mathbf{Z}^{n}$ is $\prod_{i=1}^{n}\left|F \gamma_{i}\right|$. 
The first part of this theorem is rather surprising when $F=\mathbf{H}$. Compare it to the unstable situation described in Theorem 2.3 and Proposition 2.4.

The next result describes some special elements in $D(n, F)$.

THEOREM 3.4. (i) The group $D(n, F)$ has a basis $\left\{F v_{q} \mid q=1, \ldots, n\right\}$ where $\mathbf{C} v_{q}=$ $\left(q, q^{2}, \ldots, q^{n}\right)$ and

$$
\mathbf{H} v_{q}= \begin{cases}\left(q^{2}, q^{4}, \ldots, q^{2 n}\right) & \text { if } q \text { is odd }, \\ \left(2 q^{2}, 2 q^{4}, \ldots, 2 q^{2 n}\right) & \text { if } q \text { is even }\end{cases}
$$

(ii) Let $F L_{n}$ denote the smallest positive integer $\lambda$ such that $(\lambda, 0, \ldots, 0) \in D(n, F)$. Then $\mathbf{C} L_{n}=1$. c.m. $(1,2, \ldots, n)$ and

$$
\mathbf{H} L_{n}=1 . \text { c.m. }\left\{\frac{\left|\mathbf{H} \gamma_{k}\right|}{(k-1) !(k-1) !} \mid k=1,2, \ldots, n\right\} .
$$

Since the basis just described for $D(n, \mathbf{C})$ can be realized by self maps of $\mathbf{C} P^{n}$, (ii) of Theorem 1 follows at once.

The next result describes some features of $D(n, \mathbf{H})$ which are related to the problem studied in $\S 2$.

THEOREM 3.5. (i) The vector $\left(\lambda, \lambda^{2}, \ldots, \lambda^{n}\right)$ is in $D(n, \mathbf{H})$ if and only if the integer $\lambda$ is a solution to each of the congruences $C_{1}, C_{2}, \ldots, C_{n}$ described in Theorem 2.3.

(ii) The vectors just described generate $D(n, \mathbf{H})$ if and only if $n<4$.

The first part of this theorem may be regarded as the stable verification of Feder and Gitler's conjecture. However we do not claim that the maps in (i) can necessarily be desuspended to $\mathbf{H} P^{n}$. (See Theorem 2.3 and the remarks which follow it.) The second part of Theorem 3.5 completes the proof of Theorem 1.

The proofs of these last three theorems exploit the close relationship between the group $D(n, F)$ and the appropriate $K$-theory of $F P^{n}$. To see the connection, recall that there are homotopy equivalences

$$
Q\left(\mathbf{C} P^{\infty}\right) \simeq B U \times F^{\prime}
$$

by G. Segal [25], and

$$
Q\left(\mathbf{H} P^{\infty}\right) \simeq B S p \times F^{\prime \prime}
$$

by J. Becker [6], where $Q(\quad)=\lim \Omega^{n} \Sigma^{n}(\quad)$. In these splittings, the spaces $F^{\prime}$ and $F^{\prime \prime}$ have finite homotopy groups.

Let $G=U$, or $S p$, for $F=\mathbf{C}$, or $\mathbf{H}$, respectively. V. Snaith [27] has shown that in each of the above splittings, there is a splitting map

$$
\tau: B G \rightarrow Q\left(F P^{\infty}\right)
$$

which is an $H$-map with respect to the Whitney sum on the domain and the loop multiplication on the range.

THEOREM 3.6. There are group isomorphisms

$$
\widetilde{K U}\left(\mathbf{C} P^{n}\right) \stackrel{\sim}{\rightarrow} D(n, \mathbf{C})
$$


and

$$
\widetilde{K S p}\left(\mathbf{H} P^{n}\right) \stackrel{\simeq}{\rightarrow} D(n, \mathbf{H})
$$

given by $x \rightarrow \delta$ (adjoint $\tau \circ f_{x}$ ) where $f_{x}: F P^{n} \rightarrow B G$ classifies $x$ and the cellular approximation theorem has been used, but not mentioned, to factor the indicated adjoint through the standard inclusion.

This completes the statement of results in this section.

Proof of Theorem 3.1. Let $P^{n}$ denote real projective $n$-space and assume that $n \neq 3$ or 7 . By Theorem 2.1 it suffices to show that each self map of $\Sigma^{k} P^{n}$ induces either the identity or the zero map in $\mathbf{Z} / 2$-cohomology. Perhaps the easiest way to show this, when $n$ is not of the form $2^{r}-1$, is to use the Steenrod squares. In this case each nonzero class $x_{m} \in \tilde{H}^{k+m}\left(\Sigma^{k} P^{n} ; \mathbf{Z} / 2\right)$ is linked by the Steenrod algebra to the bottom class $x_{1}$ in the following sense; there is a sequence of classes $x_{1}=$ $y_{1}, y_{2}, \ldots, y_{s}=x_{m}$ such that either $S q^{t} y_{i}=y_{i+1}$ or $S q^{t} y_{i+1}=y_{i}$ for each $i$ and some $t$ which depends on $i$. (This assertion can be verified using the formulas in [29, p. 5].) Hence for these values of $n$, Theorem 3.1 is a consequence of the action of $Q(2)$ on $H^{*}\left(P^{n} ; \mathbf{Z} / 2\right)$ and naturality.

Now assume that $n=2^{r}-1$ where $r>3$. Consider the commutative diagram

$$
\begin{array}{ccccc}
S^{n+k-1} & \stackrel{\gamma}{\rightarrow} & \Sigma^{k} P^{n-1} & \stackrel{i}{\rightarrow} & \Sigma^{k} P^{n} \\
\downarrow \lambda \iota & & \downarrow f_{n-1} & & \downarrow f \\
S^{n+k-1} & \stackrel{\gamma}{\rightarrow} & \sum^{k} P^{n-1} & \stackrel{i}{\rightarrow} & \Sigma^{k} P^{n}
\end{array}
$$

in which the rows are cofibration sequences. The attaching map $\gamma$ is essential, although the Steenrod squares now fail to detect this. That $\gamma$ is essential follows from results of James [13] and Atiyah [5] on truncated projective spaces and from Adams' solution to the vector fields on spheres problem [1].

To show that $f^{*}$ has the required form it suffices to show that the $(\bmod 2)$ reduction of $\lambda$ equals the eigenvalue of $f_{n-1}^{*}$. This tentative equality will remain unchanged if we replace the maps involved by their $q$-fold iterates, for any positive integer $q$. With this in mind, we note that the group $\left[\Sigma^{k} P^{n-1}, \Sigma^{k} P^{n-1}\right]$ is finite. A simple counting argument then shows that some iterate of $f_{n-1}$ is an idempotent. However the only idempotents in this group are the identity map and the trivial map. Indeed, any other idempotent would split off a nontrivial retract of $\Sigma^{k} P^{n-1}$. This in turn would contradict the description, given above, of the action of the Steenrod algebra on $H^{*}\left(\Sigma^{k} P^{n-1} ; \mathbf{Z} / 2\right)$.

Without loss of generality we may therefore assume that $f_{n-1}$ is either the identity or the constant map. The curresponding parity of $\lambda$ now follows using the fact that $\gamma$ is essential of order 2.

Proof of Theorem 3.6. In this proof, let $P^{n}=\mathbf{C} P^{n}$ or $\mathbf{H} P^{n}$ and let $G=U$ or $S p$, accordingly. Let $\pi: Q\left(P^{\infty}\right) \rightarrow B G$ denote a left inverse for the splitting map $\tau$. Since the fiber of $\pi$ has the rational homotopy type of a point, the composition $\tau \circ \pi$ induces the identity in rational homology. 
Let $\Phi$ denote the composite homomorphism,

$$
\left[\Sigma^{k} P^{n}, \Sigma^{k} P^{n}\right] \rightarrow\left[\Sigma^{k} P^{n}, \Sigma^{k} P^{\infty}\right] \rightarrow\left[P^{n}, Q\left(P^{n}\right)\right],
$$

which is induced by the usual inclusions $P^{n} \rightarrow P^{\infty}$ and $\Omega^{k} \Sigma^{k}\left(P^{\infty}\right) \rightarrow Q\left(P^{\infty}\right)$, and by the adjoint operator. Since $k$ is assumed to be large, the cellular approximation theorem and the suspension theorem imply that $\Phi$ is an isomorphism.

For $g \in\left[\Sigma^{k} P^{n}, \Sigma^{k} P^{n}\right]$, the assignment $g \rightarrow \pi \circ \Phi(g)$ induces an inverse to the homomorphism defined in Theorem 3.6. To see this, notice that $\Phi(g)$ and $\tau \circ \pi \circ \Phi(g)$ induce the same map in rational homology. The space $Q\left(P^{\infty}\right)$ has the rational homotopy type of a product of Eilenberg-MacLane spaces and so these two maps are rationally homotopic. This implies $g$ and $\Phi^{-1} \circ \tau \circ \pi \circ \Phi(g)$ are also rationally homotopic. Since $H_{*}\left(P^{n} ; \mathbf{Z}\right)$ is torsion free, the two maps represent the same element in $D(n, F)$. This proves Theorem 3.6.

ProOFs OF THEOREMS 3.2-3.4. We shall first consider the stable orders $\left|\gamma_{n}\right|$ of the attaching maps for complex and quaternionic projective spaces. The notation $F \gamma_{n}$ will be used only when there is a distinction to be made between the two cases.

The unstable order of $\gamma_{n}$ (i.e., the order of $\gamma_{n}$ in $\pi_{d n-1} P^{n-1}$ ) is infinite when $n>1$. This can be verified using the long exact sequence of homotopy groups induced by the fibration $\gamma$. However, for $k \geqslant 1$, the $k$-fold suspension $\Sigma^{k} \gamma_{n} \in \pi_{d n+k-1} \Sigma^{k} P^{n-1}$ is easily seen to have finite order. By the suspension theorem, this finite order is fixed once $k>d(n-2)+1$.

Lower bounds for $\left|\gamma_{n}\right|$ can be obtained using the Adams $e$-invariant $[\mathbf{2}, \mathbf{1 0}]$. In particular, the values $e_{\mathbf{C}}\left(\mathbf{C} \gamma_{n}\right)=\{1 / n !\}$ and $e_{\mathbf{C}}\left(\mathbf{H} \gamma_{n}\right)=\{2 /(2 n) !\}$ in $\mathbf{Q} / \mathbf{Z}$ can be established using the Chern character of the appropriate Hopf bundle. This implies that $\left|\mathbf{C} \gamma_{n}\right| \geqslant n$ ! and $\left|\mathbf{H} \gamma_{n}\right| \geqslant(2 n) ! / 2$. For $n$ even, we can increase this lower bound on $\left|\mathbf{H} \gamma_{n}\right|$ by a factor of 2 , since the image of $\tilde{K} S p\left(S^{8 q}\right)$ in $\tilde{K}\left(S^{8 q}\right)$ has index 2 .

The next result shows that sharp upper bounds on $\left|\gamma_{n}\right|$ can be realized after just one suspension!

Proposition 3.7. The order of $\Sigma F \gamma_{n} \in \pi_{d n} \Sigma F P^{n-1}$ is at most $n !$ for $F=\mathbf{C}$ and at most $(2 n)$ ! for $F=\mathbf{H}$.

Proof. Since there is a map $\mathbf{C} P^{\infty} \rightarrow \mathbf{H} P^{\infty}$ which is surjective in homology, it suffices to show that for each $n$ there is a map $S^{2 n+1} \rightarrow \Sigma \mathbf{C} P^{\infty}$ with degree $n$ ! in dimension $2 n+1$. Take the map $f: \Omega S^{3} \rightarrow \mathbf{C} P^{\infty}$ which classifies a 2-dimensional generator. A simple calculation shows that, for each $n, f_{*}$ has degree $n$ ! in dimension $2 n$. Now suspend once. The composition

$$
\bigvee_{n=1}^{\infty} S^{2 n+1} \simeq \Sigma \Omega S^{3} \stackrel{\Sigma f}{\rightarrow} \Sigma \mathrm{C} P^{\infty}
$$

is the required map.

This leaves one case unproved in Theorem 3.2; that of $\left|\mathbf{H} \gamma_{n}\right|, n$ odd. We shall return to this case after we have established the connection between these orders and the index of $D(n, F)$ in $\mathbf{Z}^{n}$.

We now begin the proof of Theorem 3.3. The surjection $D(n+1, F) \rightarrow D(n, F)$ in (i) is a consequence of Theorem 3.6 and the corresponding result in $K$-theory. 
Let $g: \Sigma^{k} P^{n} \rightarrow \Sigma^{k} P^{n}$ be a map with $\delta_{i}(g)=0$ for $i<m$. Using the maps $\Phi$ and $\pi$ that were defined in the proof of Theorem 3.6, let $\bar{g}=\Phi^{-1} \circ \tau \circ \pi \circ \Phi(g)$. Notice that $\delta_{i}(\bar{g})=\delta_{i}(g)$. Now $\bar{g}$ is null homotopic on $\Sigma^{k} P^{m-1}$ and so it factors:

$$
\bar{g}: \Sigma^{k} P^{n} \rightarrow \Sigma^{k} P^{n} / P^{m-1} \rightarrow \Sigma^{k} P^{n} .
$$

This shows that in $H_{k+d m}$, the image of $\bar{g}_{*}$, is generated by spherical class. A standard diagram chase shows that in this dimension the order of the cokernel of the Hurewicz homomorphism is $\left|\gamma_{m}\right|$. Hence $\left|\gamma_{m}\right|$ divides $\delta_{m}(g)$.

Take a basis for $D(n, F)$. This basis forms an $n \times n$-matrix whose determinant equals the index of $D(n, F)$ in $\mathbf{Z}^{n}$. Using row operations, this matrix can be made upper triangular. The rows in this triangular array correspond to the maps just considered in (ii). Thus we have shown that $\prod_{i=1}^{n}\left|F \gamma_{i}\right|$ is a lower bound for the index in question.

Consider the alleged basis for $D(n, F)$ which is described in Theorem 3.4. The vectors $\left(q, q^{2}, \ldots, q^{n}\right), q=1,2, \ldots$, are in $D(n, \mathbf{C})$ since they can be realized by self maps of $\mathbf{C} \boldsymbol{P}^{n}$. An easy application of the Vandermonde determinant shows that

$$
\operatorname{det}\left|\begin{array}{c}
1,1, \ldots, 1 \\
2,4, \ldots, 2^{n} \\
\vdots \\
n, n^{2}, \ldots, n^{n}
\end{array}\right|=\prod_{k=1}^{n} k !
$$

A priori, this determinant would give us an upper bound on the index of $D(n, \mathbf{C})$ in $\mathbf{Z}^{n}$. Since it equals the lower bound already established, the complex cases of Theorems 3.3(iii) and 3.4(i) are proved.

We shall now establish a basis for $D(n, \mathbf{H})$. As mentioned earlier, the vectors $\left(q^{2}, q^{4}, \ldots, q^{2 n}\right)$, for $q$ odd, can be realized by the restrictions of self maps of $\mathbf{H} P^{\infty}$ first constructed by Sullivan. For $q$ even, the vectors $\left(2 q^{2}, 2 q^{4}, \ldots, 2 q^{2 n}\right)$ can be realized as stable maps which factor through $\Sigma^{k} \mathbf{C} P^{2 n+1}$. In more detail, the fiber bundle

$$
S^{2} \rightarrow \mathbf{C} P^{2 n+1} \stackrel{p}{\rightarrow} \mathbf{H} P^{n}
$$

has a transfer [3], which can be realized by a stable map $\zeta: \Sigma^{k} \mathbf{H} P^{n} \rightarrow \Sigma^{k} \mathbf{C} P^{2 n+1}$. The composition $\Sigma^{k} p \circ \zeta$ induces multiplication by 2 on $\tilde{H}_{*} \Sigma^{k} \mathbf{H} P^{n}$. The vectors in question are then realized by the composition $\Sigma^{k} p \circ \Sigma^{k} f \circ \zeta$, where $f$ is the appropriate self map of $\mathbf{C} P^{2 n+1}$. Again using the Vandermonde determinant it follows that

$$
\operatorname{det}\left|\begin{array}{c}
\mathbf{H} v_{1} \\
\mathbf{H} v_{2} \\
\vdots \\
\mathbf{H} v_{n}
\end{array}\right|=\prod_{k=1}^{n} \frac{(2 k) !}{\varepsilon(k)} \quad \text { where } \varepsilon(k)= \begin{cases}2 & \text { if } k \text { is odd, } \\
1 & \text { if } k \text { is even. }\end{cases}
$$

Since this upper bound on the index of $D(n, \mathbf{H})$ in $\mathbf{Z}^{n}$ equals the lower bound established earlier, the proofs of Theorems 3.2, 3.3(iii) and 3.4(i) are complete. 
To finish the proofs of Theorems 3.3 and 3.4 we shall use another model for the groups $D(n, F)$.

Proposition 3.8. There is an embedding $D(n, F) \rightarrow \mathbf{Q}[x] / I$ which, in terms of the basis described in Theorem 3.4(i), is given by

$$
\left(\lambda, \ldots, \lambda^{n}\right) \rightarrow e^{\lambda x}-1 \text { for } F=\mathbf{C}, I=\left(x^{n+1}\right),
$$

and

$$
\left(\lambda^{2}, \ldots, \lambda^{2 n}\right) \rightarrow \cosh (\lambda x)-1 \text { for } F=\mathbf{H} \text { and } I=\left(x^{2 n+1}\right) .
$$

The analytic functions in this result are, of course, regarded in terms of their Maclaurin series. By abuse of notation we shall not distinguish between these power series and their images in $\mathbf{Q}[x] / I$.

Perhaps the most elementary way to verify this result is to consider the derivatives of the functions involved. Since

$$
\left.\frac{d^{m}}{d x^{m}}\left(e^{\lambda x}-1\right)\right|_{x=0}=\lambda^{m}
$$

and

$$
\left.\frac{d^{2 m}}{d x^{2 m}}(\cosh (\lambda x)-1)\right|_{x=0}=\lambda^{2 m},
$$

it is clear $D(n, \mathbf{C})$ can be identified with the $\mathbf{Z}$-linear span in $\mathbf{Q}[x] / I$ of $\left\{e^{\lambda x}-1 \mid\right.$ $\lambda=1,2, \ldots, n\}$ and that $D(n, \mathbf{H})$ corresponds to the subgroup of $\mathbf{Q}[x] / I$ which is generated by $\cosh (\lambda x)-1$ (if $\lambda$ is odd) and by $2 \cosh (\lambda x)-2$ (if $\lambda$ is even).

The reason for introducing these models of $D(n, F)$ is that they provide us with some useful identities. Consider first the complex case. Since $e^{k x}=\left(e^{x}\right)^{k}$, it follows from the binomial theorem that

$$
\left(e^{x}-1\right)^{m}=\sum_{k=1}^{m}(-1)^{m-k}\left(\begin{array}{c}
m \\
k
\end{array}\right)\left(e^{k x}-1\right) .
$$

The left side of this identity evidently corresponds to a class in $D(n, \mathbf{C})$. If

$$
\lambda_{i}=\left.\frac{d^{i}}{d x^{i}}\left(e^{x}-1\right)^{m}\right|_{x=0}, \quad i=1,2, \ldots,
$$

it is straightforward to see that $\lambda_{i}=0$ for $i<m$ and that $\lambda_{m}=m$ !. Moreover, repeated use of the chain rule shows that $m$ ! divides the integer $\lambda_{i}$ if $i \geqslant m$. Since this is true for each $m \leqslant n$, Theorem 3.3(ii) follows in the complex case.

The facts just cited imply that the functions $\left(e^{x}-1\right)^{m}, m=1,2, \ldots, n$, provide us with another basis for $D(n, \mathbf{C})$. In view of this, consider the identity

$$
x=\sum_{i=1}^{\infty} \frac{(-1)}{i}^{i-1}\left(e^{x}-1\right)^{i} .
$$

This identity may be verified by showing directly that both sides satisfy the conditions $f(0)=0$ and $f^{\prime}(x)=1$. In Theorem 3.4(ii) we seek the smallest positive $\lambda$ such that $(\lambda, 0, \ldots, 0) \in D(n, \mathbf{C})$. The answer can be described in terms of the 
identity just given. It is the smallest positive integer $\lambda$ such that when both sides of this identity are multiplied by $\lambda$, the first $n$ coefficients on the left side are integers. Clearly this number is l.c.m. $(1,2, \ldots, n)$.

The proofs in the quaternionic case are similar, but the corresponding identities are more complicated. We claim that for each $m \geqslant 1$ there is an identity,

$$
(2 \cosh (x)-2)^{m}=\sum_{k=1}^{m} a_{k}(2 \cosh (k x)-2),
$$

in which each of the coefficients $a_{k}$ are integers. Moreover if $m$ is odd, then $a_{k}$ is even for each even $k$.

To see this, let $g_{k}=2 \cosh (k x)-2$ for each $k \geqslant 0$. The basic addition formulas for the hyperbolic cosine imply the equation

$$
g_{k} g_{1}=g_{k+1}+g_{k-1}-2 g_{k}-2 g_{1}
$$

for each $k \geqslant 1$. The required identity can now be proved using induction on $m$; start with

$$
\left(g_{1}\right)^{2}=g_{2}-4 g_{1},
$$

multiply both sides by $g_{1}$, and use the above equation to simplify the right-hand side. By working $(\bmod 2)$, one sees immediately that the $a_{2 k}$ 's are even when $m$ is odd.

Thus we conclude that in $D(n, \mathbf{H})$ there are classes which correspond, by Proposition 3.8, to the functions $(2 \cosh (x)-2)^{m}$ if $m$ is even, and $\frac{1}{2}(2 \cosh (x)-2)^{m}$ if $m$ is odd. By computing the derivatives of these functions, we see that they correspond to classes of the form $\left(0, \ldots, 0, \lambda_{m}, \ldots, \lambda_{n}\right)$ where $\left|\mathbf{H} \gamma_{m}\right|$ equals $\lambda_{m}$ and divides $\lambda_{i}$ for $i>m$. A determinant calculation and Theorem 3.3(iii) shows that these classes form a basis for $D(n, \mathbf{H})$.

The proof of Theorem 3.4(ii) in the quaternionic case uses the identity

$$
\frac{x^{2}}{2}=\sum_{k=1}^{\infty}(-1)^{k} \frac{(k-1) !(k-1) !}{(2 k) !}(2 \cosh (x)-2)^{k},
$$

which is straightforward, but tedious, to verify. Here one seeks the smallest positive integer $\lambda$ such that when both sides of the above identity are multiplied by $\lambda$, the resulting coefficients on $(2 \cosh (x)-2)^{k}, k$ even, and on $\frac{1}{2}(2 \cosh (x)-2)^{k}, k$ odd, are integers for $k \leqslant n$. The answer is the least common multiple described in the statement of this theorem.

Proof of Theorem 3.5. The first part requires a closer look at the proof of Theorem 3.6. The retraction used there, $\pi: Q\left(\mathbf{H} P^{\infty}\right) \rightarrow B S p$, is the canonical extension of the map $\mathbf{H} P^{\infty} \rightarrow B S p$, which classifies $H-1 \in K S p\left(\mathbf{H} P^{\infty}\right)$ [27, Theorem 3.2]. Here $H$ denotes the canonical symplectic line bundle over $\mathbf{H} P^{\infty}$ (i.e., the Hopf bundle). Let $H_{n}$ denote the restriction of this bundle to $\mathbf{H} P^{n}$. If $f$ is a self map of $\mathbf{H} P^{n}$, it follows that the homomorphism $\pi \circ \Phi:\left[\Sigma^{k} \mathbf{H} P^{n}, \Sigma^{k} \mathbf{H} P^{n}\right] \rightarrow K S p\left(\mathbf{H} P^{n}\right)$ takes $\Sigma^{k} f$ to $f^{*}\left(H_{n}-1\right)$.

At this point it is more convenient to use complex $K$-theory. The image of $\widetilde{K S} p\left(\mathbf{H} P^{n}\right) \rightarrow \tilde{K}\left(\mathbf{H} P^{n}\right)$ is spanned by the classes $z, 2 z^{2}, z^{3}, 2 z^{4}, \ldots,\left(z^{n+1}=0\right)$, 
where $z$ denotes the image of $H_{n}-1$. Feder and Gitler showed that if $f$ has degree $\lambda$ in dimension 4 , then

$$
f^{*} z=\lambda z+\frac{\lambda(\lambda-1)}{24}\left(2 z^{2}\right)+\frac{\lambda(\lambda-1)(\lambda-4)}{360} z^{3}+\ldots
$$

The congruences $\left\{C_{k}\right\}_{1}^{n}$ arise from the requirement that the indicated coefficients are integers.

Thus we have shown that if $\left(\lambda, \lambda^{2}, \ldots, \lambda^{n}\right) \in D(n, \mathbf{H})$ and if $\lambda$ is $n$-realizable then the corresponding class in complex $K$-theory is given by the right side of the above formula. The proof of (i) will follow once we show that the extra requirement " $\lambda$ is $n$-realizable" can be omitted.

Rationalize the spaces $\mathbf{H} P^{n}, B S p$ and $B U$. This has the effect of tensoring the sequence

$$
D(n, \mathbf{H}) \stackrel{\simeq}{\rightarrow} \tilde{K} S p\left(\mathbf{H} P^{n}\right) \rightarrow \tilde{K}\left(\mathbf{H} P^{n}\right)
$$

with the rationals. Since every integer is $n$-realizable on $\mathbf{H} P_{(0)}^{n}$, the preceding formula is valid for every vector of the form $\left(\lambda, \lambda^{2}, \ldots, \lambda^{n}\right)$ in $D(n, \mathbf{H}) \otimes \mathbf{Q}$. Among these vectors, those which live in $D(n, \mathbf{H})$ are precisely those whose image is integral in $\tilde{K}\left(\mathbf{H} P^{n}\right) \otimes \mathbf{Q}$. This completes the proof of (i).

The proof of (ii) requires a little arithmetic. As was mentioned earlier, the integrality conditions of Theorem 2.3 are known to imply $n$-realizability for $n<4$. Here then are some $n$-realizable integers:

$$
\begin{array}{ll}
n=1, & \lambda=1 ; \\
n=2, & \lambda=1,9,24 ; \\
n=3, & \lambda=1,9,16,25,40 .
\end{array}
$$

We leave it to the reader to show that $D(n, \mathbf{H})$ is generated by vectors of the form $\left(\lambda, \lambda^{2}, \ldots, \lambda^{n}\right)$ for the values of $n$ and $\lambda$ just given.

Suppose that vectors of this form generate $D(n, \mathbf{H})$ for $n \geqslant 4$. Then by Theorems 3.2 and 3.3(ii), the vector $(0,0,360, ?, \ldots, ?)$ can be written as a linear combination of them. This implies the existence of equations

$$
\sum_{1}^{m} a_{i} \lambda_{i}=0, \quad \sum_{1}^{m} a_{i} \lambda_{i}^{3}=360
$$

and

$$
\sum_{1}^{m} a_{i}\left(\lambda_{i}^{3}-\lambda_{i}\right)=360
$$

for some set of solutions $\left\{\lambda_{i}\right\}_{1}^{m}$ to $C_{2}, C_{3}$ and $C_{4}$, and integers $a_{1}, \ldots, a_{m}$. But for these solutions it is easy to check that either $\nu_{2}\left(\lambda_{i}\right) \geqslant 5$ or $\nu_{2}\left(\lambda_{i}-1\right) \geqslant 3$, where $\nu_{2}(q)$ denotes the highest power of 2 which divides $q$. In either event, this implies that $\nu_{2}\left(\lambda_{i}^{3}-\lambda_{i}\right) \geqslant 4$. This is a contradiction since $\nu_{2}(360)=3$.

4. Applications. Our first application deals with fixed points. In view of the Lefschetz fixed point theorem [28, p. 195], those spaces for which every self map has a nonzero Lefschetz number (and hence a fixed point) are of some interest. 
Projective spaces, and their suspensions, provide a rich source of such spaces. Using the results in $\S 2$, it follows that every self map of $P^{n}$ has a nonzero Lefschetz number if and only if $n$ is even in the real and complex cases or $n \geqslant 2$ in the quaternionic case.

In the following result assume that $k$ is large with respect to $n$. We use Theorem 3.4 to prove this rather amusing stable result.

THEOREM 4.1. Each self map of $\Sigma^{k} F P^{n}$ has a nonzero Lefschetz number if and only if $n$ is even and $F=\mathbf{R}, \mathbf{C}$ or $\mathbf{H}$, or $n$ is odd, $F=\mathbf{H}$, and $p^{2}-p$ divides $2 n$, for some odd prime $p$.

The next three applications involve the notion of the genus set of homotopy type. Recall from [12] that for a nilpotent space $X$ of finite type, the genus set $G(X)$ is the set of homotopy types $Y$ such that:

(i) $Y$ is nilpotent,

(ii) $Y$ has finite type, and

(iii) $Y_{(p)} \simeq X_{(p)}$ for all primes $p$.

Before describing our results, we recall that the space $\mathbf{R} P^{2 n}$ is not nilpotent $[28, \mathbf{p}$. 420]. Therefore this space is excluded from the following remarks about $G\left(P^{n}\right)$.

In general, the only structure on $G(X)$ appears to be that of a filtered set; $F_{n} G(X)=\{Y \in G(X) \mid Y$ has the same $n$-type as $X\}$. However when $X$ is a projective space or the stable suspension of one, it is possible to give $G(X)$ the structure of a filtered abelian group.

In this case the group operation is defined as follows; if $Y_{1}$ and $Y_{2}$ are in $G(X)$, then their sum $Y_{1}+Y_{2}$ is represented by a pullback of maps:

$$
\begin{aligned}
& Y_{2} \\
& \downarrow f_{2} \\
& Y_{1} \stackrel{f_{1}}{\rightarrow} \quad X
\end{aligned}
$$

Both maps here are required to be equivalences at each prime divisor of a certain integer $t=t(X)$. For any other prime $p$, either $f_{1}$ or $f_{2}$ is to be an equivalence at $p$.

It is not obvious that this group operation is well-defined. That is is well-defined is one of the many remarkable features of a theorem due to Zabrodsky [33], which relates the self maps of certain spaces to their genus. We use this theorem, stated as Theorem 4.6, and the results of $\$ 2$ to prove the following one.

THEOREM 4.2. For $1 \leqslant n<\infty$,

(i) $G\left(\mathbf{R} P^{2 n+1}\right)=1$ and $G\left(\mathbf{R} P^{2 n}\right)$ is not defined,

(ii) $G\left(\mathbf{C} P^{n}\right)=1$,

(iii) $G\left(\mathbf{H} P^{n}\right) \simeq \mathbf{Z} / 2 \oplus \cdots \oplus \mathbf{Z} / 2$, where the number of factors equals the number of primes $p$ such that $2 \leqslant p \leqslant 2 n-1$.

Here we write $G(X)=1$ if $X$ is the only member of its genus. The quaternionic result was suggested by Rector's calculation of $G\left(\mathbf{H} P^{\infty}\right)$ [22], and is probably a simple consequence of his result. However we think that the reader may be amused at our use of Zabrodsky's machine in proving (iii). 
Since localization commutes with suspension, there is an obvious inclusion $G(X)$ $\rightarrow G(\Sigma X)$. For a space $X$, define its stable genus $G S(X)=\lim _{\rightarrow} G\left(\Sigma^{k} X\right)$.

TheOREM 4.3. For $1 \leqslant n<\infty, G S\left(\mathbf{R} P^{n}\right)=1$. For $F=\mathbf{C}$ or $\mathbf{H}$,

$$
G S\left(F P^{n}\right) \simeq \prod_{m=1}^{n}\left(\mathbf{Z} /\left|F \gamma_{m}\right|\right)^{*} / \pm 1
$$

In this theorem, $\left|F \gamma_{m}\right|$ refers to the stable orders that were computed in Theorem 3.2 and $(\mathbf{Z} / q)^{*}$ denotes the group of units in the ring of integers $\bmod q$. The filtration on the genus set, which was mentioned earlier, plays an important role in the proof of Theorem 4.3. We show that $G S\left(F P^{n}\right)$ is isomorphic to the graded group associated with this filtration.

The next result involves the Lie group $S U(n)$. The connection between this group and projective spaces is the following: there is a well known embedding $i$ : $\Sigma \mathbf{C} P^{n-1}$ $\rightarrow S U(n)$ which takes $H_{*} \Sigma \mathrm{C} P^{n-1}$ isomorphically onto the module of primitives $\mathrm{PH}_{*} \mathrm{SU}(n)$ [29, p. 39]. Moreover James has shown that there is a left inverse $r: \Sigma^{\infty} S U(n) \rightarrow \Sigma^{\infty}\left(\Sigma C P^{n-1}\right)$ for $\Sigma^{\infty}(i)[14$, p. 50].

THEOREM 4.4. There is an epimorphism $G(S U(n)) \rightarrow G S\left(\mathbf{C} P^{n-1}\right)$. With respect to Zabrodsky's description of the genera of these spaces in terms of their self maps (Theorem 4.6 and Corollary 4.7), this epimorphism is included by the operation which sends each self map $f$ of $S U(n)$ to the self map $r \circ \Sigma^{\infty}(f \circ i)$ of $\Sigma^{\infty} \mathbf{C} P^{n-1}$.

This epimorphism and the previous theorem imply the following estimate which is stated in terms of the Euler $\phi$-function

$$
|G(S U(n))| \geqslant \prod_{m=1}^{n-1}[\phi(m !) / 2] .
$$

This lower bound was first proved by Zabrodsky in [33, p. 152]. There he conjectured that this estimate is in fact an equality. This conjecture, if true, would imply a connection between $\mathbf{C} P^{n-1}$ and $S U(n)$ that goes far beyond the one cited above.

Our final application concerns the $2 n-1$ connective cover of the sphere $S^{2 n-1}$. Denote this space by $S^{2 n-1}\langle 2 n-1\rangle$ and assume that it has been localized at a prime $p$. One can then ask, for what values of $k$ does the identity map on the $k$-fold loop space $\Omega^{k} S^{2 n-1}\langle 2 n-1\rangle$ have finite order? Here the order of the identity map means the smallest positive integer $m$ such that the power map $x \rightarrow x^{m}$ is null homotopic.

It is known, for example, if $p$ is an odd prime and $k \geqslant 2 n-2$, then the order of identity map on $\Omega^{k} S^{2 n-1}\langle 2 n-1\rangle$ is exactly $p^{n-1}$. Various cases of this remarkable fact were discovered by Selick [26], Cohen, Moore and Neisendorfer [7], and Neisendorfer [20]. This geometric fact implies the celebrated result that $p^{n-1}$ annihilates the $p$-primary torsion in $\pi_{*} S^{2 n-1}$ which is also due to the four authors just named.

On the other hand, Neisendorfer and Selick have shown that if $0<k<2 n-3$, then the corresponding identity map does not have finite order [21]. We will give a slightly different proof of their result. To prove it we use Postnikov approximations of $S^{2 n-1}\langle 2 n-1\rangle$ and the results of Theorem 3.4. 
THEOREM 4.5. Let $X$ denote a space of type $S^{2 n-1}(2 n, \ldots, 2 n+2 m)$ localized at a prime $p$. Assume that $p^{f} \leqslant m+2$ and that $0<k<2 n-3$. Then, in the group $\left[\Omega^{k} X, \Omega^{k} X\right]$, the homotopy class of the identity map has order not less than $p^{f}$.

This completes our list of applications.

Proof of Theorem 4.1. For the real projecitve spaces, the proof is an immediate consequence of Theorems 2.1 and 3.1. In the other two cases, consider the homomorphism

$$
\varrho: D(n, F) \rightarrow \mathbf{Z}
$$

given by

$$
\left(\lambda_{1}, \lambda_{2}, \ldots, \lambda_{n}\right) \rightarrow \sum_{i=1}^{n} \lambda_{i} .
$$

Clearly there is a self map of $\sum^{k} F P^{n}$ with Lefschetz number zero if and only if $\mathcal{E}$ is surjective.

For $n$ even, it suffices to check the image of $\mathcal{L}$ on the basis for $D(n, F)$ given in Theorem 3.4(i). In this case $\mathcal{L}$ is not surjective; its cokernel has order $\geqslant 2$.

For $n$ odd and $F=\mathbf{C}, \mathcal{L}$ is surjective as can be seen by considering the vector $\left(-1,1, \ldots,(-1)^{n}\right)$.

In the last remaining case ( $F=\mathbf{H}, n$ odd) suppose that $\varrho$ is not surjective. By Theorem 3.4(i), it follows that there is a prime $p$ which divides $n$ and also $\mathcal{E}\left(v_{q}\right)$ for $q=2, \ldots, n$. For these values of $q$,

$$
\mathcal{L}\left(v_{q}\right)=\frac{q^{2}\left(q^{2 n}-1\right)}{q^{2}-1}
$$

up to a factor of 2 . This implies that for all $q$ relatively prime to $p, q^{2 n} \equiv 1 \bmod (p)$. Since $q$ could be chosen to generate the group of units in $\mathbf{Z} / p$, it follows that $p-1$ divides $2 n$. This argument can easily be reversed and so the proof of Theorem 4.1 is complete.

Proof of TheORem 4.2. In (i) let $P^{k}$ denote $\mathbf{R} P^{k}$ and assume that $Y$ represents a homotopy type in the genus of $P^{2 n+1}$. It follows that $\pi_{*} Y \simeq \pi_{*} P^{2 n+1}$. Let $f_{1}: P^{1} \rightarrow Y$ represent a generator of $\pi_{1} Y$. If $n=0$, then $f_{1}$ is a homotopy equivalence. If $n \geqslant 1$, there is an extension $f_{2}: P^{2} \rightarrow Y$ which induces an isomorphism on fundamental groups. Since $\pi_{q} Y=0$ for $1<q<2 n+1$, there is no obstruction to extending $f_{2}$ to a map $f: P^{2 n+1} \rightarrow Y$. Since the $Z / 2$-cohomology rings of $Y$ and $P^{2 n+1}$ are isomorphic, it follows that $f$ induces a $\mathbf{Z} / 2$-cohomology isomorphism. This implies that in integral homology $f$ has odd degree in dimension $2 n+1$. If this degree is not \pm 1 , we will consider the composition

$$
P^{2 n+1} \stackrel{p}{\rightarrow} P^{2 n+1} \vee S^{2 n+1} \stackrel{(f, g)}{\rightarrow} Y
$$

where $p$ pinches to a point the boundary of an embedded $2 n+1$-ball. Let $y \in$ $H_{2 n+1}(Y ; \mathbf{Z})$ be a generator. A standard diagram chase shows that $2 y$ is spherical. Therefore the class $g \in \pi_{2 n+1} Y$ may be chosen so that the above composition induces an integral homology isomorphism. By Dror's generalization of the 
Whitehead theorem to nilpotent spaces, [9], we have a homotopy equivalence between $Y$ and $P^{2 n+1}$.

The proof that $G\left(\mathbf{C} P^{n}\right)=1$ is very similiar to the one just given and will be omitted.

Now let $P^{n}$ denote quaternionic projective $n$-space. For any space $X$, let $X(1, \ldots, m)$ denote the Postnikov approximation whose homotopy groups are naturally isomorphic to those of $X$ up through dimension $m$, and are zero beyond this dimension.

By the results of Mimura and Toda [19], the spaces $P^{n}$ and $P^{n}(1, \ldots, 4 n)$ are $p$-universal for each prime $p$. Hence if $X$ denotes either space, then $Y \in G(X)$ if and only if for each prime $p$ there is a map $f: Y \rightarrow X$ which is an equivalence at $p$. This implies that the genus sets of $P^{n}$ and $P^{n}(1, \ldots, 4 n)$ are isomorphic. The isomorphism between them is induced by the operation $Y \rightarrow Y(1, \ldots, 4 n)$. Restriction to the $4 n$-skeleton induces the required inverse.

To compute $G\left(\mathbf{H} P^{n}\right)$ we shall use the space $P^{n}(1, \ldots, 4 n)$, the maps in Proposition 2.4(i), and a theorem of Zabrodsky. This theorem requires some special hypotheses and definitions which we shall now describe.

Let $X$ be a simply connected space of finite type with only finitely many nonzero homotopy groups. Assume that its rationalization $X_{0}$ is an $H$-space and let $n_{1}<n_{2}$ $<\cdots<n_{\text {l }}$ denote those dimensions $n$ for which $\pi_{n} X_{0} \neq 0$. For each self map $f$ of $X$, define

$$
d_{i}(f)=\operatorname{determinant}\left(f_{0}\right)_{\#}: \pi_{n} X_{0} \leftrightarrow \quad \text { for } i=1, \ldots, l \text {. }
$$

For any integer $t \geqslant 2$, define

$$
\mathcal{E}(X, t)=\left\{f \in[X, X] \mid f_{(p)}: X_{(p)} \stackrel{\sim}{\rightarrow} X_{(p)} \text { if } p \text { divides } t\right\} .
$$

TheOREM 4.6 (ZABRODSKY [32]). For $X$ just described, there is a positive integer $t$ which depends on $X$ and an exact sequence

$$
\mathcal{E}(X, t) \stackrel{d}{\rightarrow}\left((\mathbf{Z} / t)^{*} / \pm 1\right)^{l} \rightarrow G(X) \rightarrow 0
$$

where $d(f)=\left(d_{1}(f), \ldots, d_{l}(f)\right)$.

By abuse of notation, $d_{i}(f)$ denotes also the image of $d_{i}(f)$ in the group $(\mathbf{Z} / t)^{*} / \pm 1$.

We need to know something about the number $t$. Zabrodsky defines it as follows. First recall that the exponent of a finite abelian group $G$ is the smallest positive integer $m$ such that $m x=0$ for all $x \in G$. Let

$a_{j}=$ the exponent of the torsion subgroup of $\pi_{j} X$, and

$b_{j}=$ the exponent of the cokernel of the Hurewicz-Serre homomorphism $\pi_{j}(X) \rightarrow$ $P H_{j}(X ; \mathbf{Z}) /$ torsion.

Assume that $\pi_{n} X=0$ for $n \geqslant N$. Then $t=t(X ; N)$ is the least common multiple of $\prod_{j=1}^{N} a_{j} b_{j}$ and the torsion primes of $H_{m}(X ; \mathbf{Z})$ and $Q H^{m}(X ; \mathbf{Z})$, where $m \leqslant N$.

Now let $X=P^{n}(1, \ldots, 4 n)$. We have $l=1$ since $X_{0} \simeq K(\mathbf{Q}, 4)$. The isomorphism $\pi_{j} X \simeq \pi_{j-1} S^{3}$ for $j \leqslant 4 n$ and Serre's classical results on $\pi_{*} S^{3}[29$, p. 90] imply that 
we may take $t=\prod_{i=1}^{k} p_{i}^{n_{i}}$ where the $p_{i}$ 's run through all primes $\leqslant 2 n-1$. A simple application of the Euler $\phi$-function then shows that the order

$$
\left|(\mathbf{Z} / t)^{*} / \pm 1\right|=\frac{t}{2} \prod_{i=1}^{k}\left(1-\frac{1}{p_{i}}\right) .
$$

Since $[X, X] \approx\left[P^{n}, P^{n}\right]$, the order of the subgroup $d(\mathcal{E}(X, t))$ can be calculated using Proposition 2.4(i). It equals the number of integers $\lambda$ between 0 and $t$ such that $\lambda \equiv 1 \bmod 8$ and $0 \not \lambda \equiv x^{2} \bmod p$ for each odd prime $p<2 n$. By the Chinese remainder theorem this number is

$$
|d(\mathcal{E}(X, t))|=\frac{t}{2} \prod_{i=1}^{k} \frac{1}{2}\left(1-\frac{1}{p_{i}}\right) .
$$

The order of the quotient $G(X)$ is thus $2^{k}$ as claimed. It is clear that this group has exponent 2 since for any $x \in(\mathbf{Z} / t)^{*}, x^{2}$ is in the image of $d$.

Proof of Theorem 4.3. In the stable category there is a dual version of Theorem 4.6. We shall use this dual version and the results of $\S 3$ to compute the stable genus of a projective space.

Let $K$ be a connected finite complex in the stable range (i.e., its dimension is less than twice its connectivity). Let $n_{1}<n_{2}<\cdots<n_{l}$ denote those dimensions $n$ for which $\tilde{H}_{n}(K ; \mathbf{Q}) \neq 0$. For a self map $f$ of $K$ let $\delta_{i}(f)$ denote the determinant of $f_{*}: H_{n_{i}}(K ; \mathbf{Q}) \hookleftarrow$.

COROllary 4.7. For $K$ just described there is a number $t$ which depends on $K$ and an exact sequence

$$
\mathcal{E}(K, t) \stackrel{\delta}{\rightarrow}\left((\mathbf{Z} / t)^{*} / \pm 1\right)^{l} \rightarrow G(K) \rightarrow 0
$$

where $\delta(f)=\left(\delta_{1}(f), \ldots, \delta_{l}(f)\right)$.

Proof. We may assume the suspension $E: K \rightarrow \Omega \Sigma K$ is an $N$-equivalence where $N$ exceeds the dimension of $K$. It follows that the genus sets of $K$ and $\Omega \Sigma K(1, \ldots, N)$ are isomorphic, since both spaces are $p$-universal for all primes $p$. Apply Zabrodsky's theorm to the latter space and notice that $\delta_{i}(f)=d_{i}(\Omega \Sigma f)$.

The above corollary is due to Davis [8], but the proof given here seems much easier than his.

Let us apply Corollary 4.7 to an appropriate suspension of $\mathbf{R} P^{n}$. In this case, $t$ is a multiple of 2 and $l=0$ or 1 . Theorem 2.1 provides us with enough self maps to conclude that $\delta$ is surjective and hence $G S\left(\mathbf{R} P^{n}\right)=1$.

The proof in the complex and quaternionic cases uses the ring structure on $D(n, F)$ which is induced by the composition of stable self maps. This ring structure makes $D(n, F)$ a subring of $\mathbf{Z}^{n}$. In terms of coordinates, the multiplication in both rings has the form

$$
\left(a_{1}, \ldots, a_{n}\right) \cdot\left(b_{1}, \ldots, b_{n}\right)=\left(a_{1} b_{1}, \ldots, a_{n} b_{n}\right) .
$$


Now fix $n$ and $F$ and let $d_{m}=\left|F \gamma_{m}\right|, m=1,2, \ldots$. We may assume that the number $t$ is a multiple of $d_{1} d_{2} \ldots d_{n}$. To simplify notation further, let

$$
\begin{aligned}
& D=(D(n, F) \otimes \mathbf{Z} / t)^{*}, \\
& Z=\left(\mathbf{Z}^{n} \otimes \mathbf{Z} / t\right)^{*}, \text { and } \\
& C=Z / D .
\end{aligned}
$$

Here, $R^{*}$ denotes the group of units in a commutative ring $R$.

It is not difficult to see the connection between the groups just defined and those which occur in the statement of Corollary 4.7. The map $\delta$ in (4.7) factors through $D$ and so $G S\left(F P^{n}\right)$ is a quotient of $C$.

We shall now compute the cokernel $C$. For $1 \leqslant m \leqslant n+1$ let $Z_{m}=\left\{\left(\lambda_{1}, \ldots, \lambda_{n}\right)\right.$ $\in Z \mid \lambda_{i}=1$ for $\left.i<m\right\}, D_{m}=D \cap Z_{m}$, and $C_{m}=Z_{m} / D_{m}$. Then $C=C_{1} \supseteq C_{2} \supseteq$ $\cdots C_{n} \supseteq C_{n+1}=1$. For each $m \leqslant n$, we have a short exact sequence

$$
0 \rightarrow D_{m} / D_{m+1} \rightarrow(\mathbf{Z} / t)^{*} \rightarrow C_{m} / C_{m+1} \rightarrow 0 .
$$

For any divisor $d$ of $t$, let

$$
K(d)=\operatorname{Ker} \rho:(\mathbf{Z} / t)^{*} \rightarrow(\mathbf{Z} / d)^{*}
$$

where $\rho$ denotes reduction $\bmod d$.

LeMma 4.8. $D_{m} / D_{m+1} \simeq K\left(d_{m}\right)$.

Proof. Let $P^{n}$ denote $\Sigma^{k} F P^{n}$ where $k$ is large. If $f: P^{n} \leftrightarrow$ represents a class in $D_{m}$ it follows from Theorem 3.3 that $\delta_{m}(f) \equiv 1 \bmod d_{m}$. This implies that $D_{m} / D_{m+1}$ is a subgroup of $K\left(d_{m}\right)$.

On the other hand, if $\lambda$ is any integer such that $\lambda \equiv 1 \bmod d_{m}$, it also follows from Theorem 3.3 that there is a self map $g$ of $P^{m}$ such that $\delta_{i}(g)=1$ for $i<m$ and $\delta_{m}(g)=\lambda$.

Therefore if $\Pi$ denotes the set of primes which divide $t$, it suffices to show that any $\Pi$-equivalence of $P^{m}$ extends (up to homology) to a $\Pi$-equivalence of $P^{n}$. We shall show this by considering one cell at a time.

Suppose $g: P^{m} \leftrightarrow$ is such an equivalence. By Theorem 3.3 we may assume that $g$ extends to some self map $\bar{g}$ of $P^{m+1}$. Thus if $\gamma$ denotes the attaching map for the top cell of $P^{m+1}$ it follows that $g_{\#}(\gamma)=\lambda \gamma$ for some integer $\lambda=\delta_{m+1}(\bar{g})$. Since $g$ is a II-equivalence and $\gamma$ has order $d_{m+1}$ it follows that $\left(\lambda, d_{m+1}\right)=1$.

If $(\lambda, t)=1$, then $\bar{g}$ is the map we want. If this is not the case let $\lambda^{\prime}=\lambda+r d_{m+1}$ where $r$ is the product of those primes which divide $t$ but do not divide $\lambda \cdot d_{m+1}$. It follows that $\left(\lambda^{\prime}, t\right)=1$. Since we can alter the degree of $\bar{g}$ on the top cell of $P^{m+1}$ by any multiple of $d_{m+1}$, we see that $g$ extends to a $\Pi$-equivalence of $P^{m+1}$. This argument can be repeated on each cell of $P^{n}$ and so the lemma is proved.

By combining Lemma 4.8 with the short exact sequence which preceded it, we obtain another;

$$
0 \rightarrow C_{m+1} \stackrel{i}{\rightarrow} C_{m} \stackrel{\pi}{\rightarrow}\left(\mathbf{Z} / d_{m}\right)^{*} \rightarrow 0 .
$$

We shall show that this sequence is split exact. This amounts to identifying in $C_{m}$ a subgroup which is taken by $\pi$ isomorphically onto $\left(\mathbf{Z} / d_{m}\right)^{*}$. To this end, let $q$ be the 
largest integer which both divides $t$ and is relatively prime to $d_{m}$. Define

$$
\begin{aligned}
& Z_{m}(q)=\left\{\left(\lambda_{1}, \ldots, \lambda_{n}\right) \in Z_{m} \mid \lambda_{i} \equiv 1 \bmod q \text { for all } i\right\}, \\
& D_{m}(q)=D \cap Z_{m}(q) \text { and } C_{m}(q)=Z_{m}(q) / D_{m}(q) .
\end{aligned}
$$

Notice that $Z_{m}(q) \simeq K(q) \times \cdots \times K(q),(n-m+1)$-times. Since $\left(q, d_{m}\right)=1$, the reduction map $\rho:(\mathbf{Z} / t)^{*} \rightarrow\left(\mathbf{Z} / d_{m}\right)^{*}$ is still an epimorphism when restricted to $K(q)$. This implies that the restriction $\pi_{q}$ in the following diagram is also surjective:

$$
\begin{array}{ccc}
C_{m} & \pi & \\
i_{q} \uparrow & \searrow & \left(\mathbf{Z} / d_{m}\right)^{*} \\
C_{m}(q) & \nearrow & \\
\pi_{q} &
\end{array}
$$

The next lemma will enable us to determine the structure of the subgroup $C_{m}(q)$.

Lemma 4.9. Let $l_{j}$ denote the least common multiple of $q$ and $d_{j}$. There is an isomorphism

$$
K\left(l_{m}\right) \times K\left(l_{m+1}\right) \times \cdots \times K\left(l_{n}\right) \simeq D_{m}(q)
$$

which for $j=m, \ldots, n$ takes $K\left(l_{j}\right)$ into $D_{j}$.

Proof. This proof is similiar to the proof of Lemma 4.8. Using the notation established there, assume that $k \geqslant m$ and that $f: P^{k} \hookleftarrow$ is a П-equivalence such that $\delta(f) \equiv(1,1, \ldots, 1) \bmod q$. We shall first show that $f$ extends, up to homology, to a self map of $P^{n}$ with the same properties.

Let $\gamma$ denote the attaching map for the top cell of $P^{k+1}$. As in Lemma 4.8 we may assume that $f_{\#} \gamma=\lambda \gamma$ where $\left(\lambda, d_{k+1}\right)=1$. In addition, the hypothesis on $\delta(f)$ implies, by Theorem 3.3(ii), that $\lambda \equiv 1 \bmod \operatorname{gcd}\left(q, d_{k+1}\right)$. A simple counting argument shows that we may choose a coefficient $c$ so that $\lambda^{\prime}=\lambda+c d_{k+1} \equiv 1 \bmod q$. Since every prime divisor of $t$ divides either $q$ or $d_{k+1}$ it follows that $\left(\lambda^{\prime}, t\right)=1$. Hence $f$ extends, up to homology, to a self map of $P^{k+1}$ with degree $\lambda^{\prime}$ on the top cell. By induction $f$ extends to a $\Pi$-equivalence $f^{\prime}$ of $P^{n}$ such that $\delta\left(f^{\prime}\right) \equiv(1,1, \ldots, 1)$ $\bmod q$.

It is clear from Theorem 3.2 that each $d_{j}$ divides $d_{j+1}$. This implies that $l_{j}$ and $t$ have the same prime divisors for $j=m, \ldots, n$. It then follows from elementary number theory (e.g., [24, Chapter 2]) that each of the kernels $K\left(l_{j}\right)$ is cyclic.

For $j=m, \ldots, n$ let $a_{j}$ be an integer whose $(\bmod t)$ reduction generates $K\left(l_{j}\right)$ and let $f_{j}: P^{n} \hookleftarrow$ be a map such that $\delta\left(f_{j}\right) \equiv(1,1, \ldots, 1) \bmod l_{j}$ and, in particular,

$$
\delta_{i}\left(f_{j}\right)= \begin{cases}1 & \text { if } i<j, \\ a_{j} & \text { if } i=j .\end{cases}
$$

By Theorem 3.3(ii) and the first part of this proof we know that such self maps of $P^{n}$ exist. The classes $\delta\left(f_{m}\right), \ldots, \delta\left(f_{n}\right)$ generate in $D_{m}(q)$ a subgroup which is evidently isomorphic to $K\left(l_{m}\right) \times \cdots \times K\left(l_{n}\right)$. A simple filtration argument then shows that the order of $D_{m}(q)$ equals the order of this subgroup and the result follows. 
From the description of $D_{m}(q)$ just obtained it follows that there is an isomorphism

$$
C_{m}(q)=Z_{m}(q) / D_{m}(q) \simeq Q_{m} \times \cdots \times Q_{n}
$$

where $Q_{j} \simeq K(q) / K\left(l_{j}\right)$ for $j=m, \ldots, n$. This isomorphism when composed with $i_{q}: C_{m}(q) \rightarrow C_{m}$ takes $Q_{j}$ into $C_{j}$. Since $q$ and $d_{m}$ are relatively prime, $l_{m}=q d_{m}$, and $Q_{m} \simeq\left(\mathbf{Z} / d_{m}\right)^{*}$. The map $\pi_{q}: C_{m}(q) \rightarrow\left(\mathbf{Z} / d_{m}\right)^{*}$ thus takes an isomorphic copy of $Q_{m}$ onto $\left(\mathbf{Z} / d_{m}\right)^{*}$.

Since the maps $\pi_{q}$ and $\pi$ are split surjective we can conclude that

$$
C_{m} \simeq\left(\mathbf{Z} / d_{m}\right)^{*} \times C_{m+1} .
$$

Since this is true for each $m$ we have

$$
C \simeq \prod_{m=1}^{n}\left(\mathbf{Z} / d_{m}\right)^{*} .
$$

The $\mathbf{Z} / 2$ action of $\{ \pm 1\}$ on the $m$ th coordinate in $Z$ passes to the quotient $C_{m} / C_{m+1}$ for each $m$, and so by Corollary 4.7 ,

$$
G S\left(F P^{n}\right) \simeq \prod_{m=1}^{n}\left(\mathbf{Z} / d_{m}\right)^{*} / \pm 1 .
$$

Proof of THEOREM 4.4. The proof centers on the following diagram:

$$
\begin{aligned}
& \mathcal{E}\left(\Sigma^{\infty} \mathbf{C} P^{n-1}, t\right) \stackrel{\delta}{\rightarrow}\left[(\mathbf{Z} / t)^{*} / \pm 1\right]^{n-1} \quad \rightarrow \quad G S\left(\mathbf{C} P^{n-1}\right) \rightarrow 0 \\
& \uparrow A \quad \uparrow I \quad \uparrow I / A \\
& \mathcal{E}(S U(n), t) \stackrel{d}{\rightarrow}\left[(\mathbf{Z} / t)^{*} / \pm 1\right]^{n-1} \quad \rightarrow \quad G(S U(n)) \rightarrow 0
\end{aligned}
$$

Here $A$ denotes the operation described in the statement of the theorem, $I$ is the identity, and $I / A$ is the induced homomorphism on quotients.

There are some items in this diagram which need to be explained and justified. Starting with the bottom row, let $X$ be a space of type $S U(n)(1, \ldots, N)$ where $\operatorname{dim} S U(n)<N<\infty$. By an argument analogous to the one used in Theorem 4.3, the genus sets of $X$ and $S U(n)$ are isomorphic. Moreover $X$ satisfies the hypothesis of Zabrodsky's Theorem 4.6. Let $t=t(X, N)$ in the diagram. It is not difficult to see that the image of $d$ coincides with the subgroup $d(\mathcal{E}(X, t))$. Therefore by Theorem 4.6, the bottom row is exact at the second and third terms.

How large is the number $t$ ? By Bott's calculations of $\pi_{*} U(n)$ we know that the Hurewicz homomorphism fits into an exact sequence

$$
0 \rightarrow \pi_{2 k+1} S U(n) \stackrel{h}{\rightarrow} P H_{2 k+1} S U(n) \rightarrow \mathbf{Z} / k ! \rightarrow 0
$$

for $k=1, \ldots, n-1$. Therefore $t$ must be a multiple of $\prod_{k=1}^{n-1} k$ !. This was the only assumption we placed on $t$ in our calculation of $G S\left(C P^{n-1}\right)$ in Theorem 4.3. Therefore the top row is also exact at the second and third terms.

It remains to show that the first square commutes. Given a self map $f$ of $S U(n)$, let $P_{*}(f)$ denote the restriction of $f_{*}$ to the module of primitives, $P \tilde{H}_{*} S U(n)$. In the following diagram the Hurewicz homomorphism $h$ is a rational isomorphism. 


$$
\begin{array}{ccc}
P \tilde{H}_{*} S U(n) & \stackrel{P}{*}(f) & P \tilde{H}_{*} S U(n) \\
\uparrow h & & \uparrow h \\
\pi_{*} S U(n) & \stackrel{f_{\#}}{\rightarrow} & \pi_{*} S U(n)
\end{array}
$$

Thus in dimension $2 k+1$, the degree of $f_{\#}$ equals that of $P_{*}(f)$, for $k=1, \ldots, n-1$.

We may regard the map $A(f)$ as the composition

$$
\Sigma^{k+1} \mathbf{C} P^{n-1} \stackrel{\Sigma^{k} i}{\rightarrow} \Sigma^{k} S U(n) \stackrel{\Sigma^{k} f}{\rightarrow} \Sigma^{k} S U(n) \stackrel{r}{\rightarrow} \Sigma^{k+1} \mathbf{C} P^{n-1}
$$

where $k$ is large and $r \circ \Sigma^{k} i$ is homotopic to the identity. Therefore, since $i_{*} H_{*} \Sigma \mathbf{C} P^{n-1}=P H_{*} S U(n)$, it follows by the naturality of the suspension isomorphism that $d(f)=\delta A(f)$.

It is now clear that the second square also commutes and that the quotient map $I / A$ is an epimorphism as claimed.

REMARK. Using ad hoc techniques I can construct enough self maps of $S U(n)$ for $n \leqslant 5$ to conclude that $G(S U(n)) \simeq G S\left(\mathbf{C} P^{n-1}\right)$ for these values of $n$. For larger values of $n$ the isomorphism question is still open.

Proof of Theorem 4.5. Assume that all spaces and maps have been localized at $p$. We shall use the cofibration sequence

$$
Y \stackrel{g}{\rightarrow} S^{2 n-1} \stackrel{i}{\rightarrow} \Sigma^{2 n-3} \mathbf{C} P^{m+2}
$$

where $Y=\Sigma^{2 \mathrm{n}-4} \mathbf{C} P^{m+2} / \mathbf{C} P^{1}$ and $0 \leqslant m<\infty$.

Let $p^{e}$ denote the order of the identity map on $\Omega^{2 n-4} X$ where $X=$ $S^{2 n-1}(2 n, \ldots, 2 n+2 m)$. The induced isomorphisms of track groups,

$$
\left[Y, S^{2 n-1}\right] \stackrel{\simeq}{\leftarrow}\left[Y, S^{2 n-1}\langle 2 n-1\rangle\right] \stackrel{\sim}{\rightarrow}[Y, X]
$$

imply that $p^{e} g=0$ in the first group.

Apply the functor $\left[, S^{2 n-1}\right]$ to the above cofibration sequence. By exactness at the group in the middle, it follows that $p^{e} \iota=i^{*}(h)$ for some map $h: \Sigma^{2 n-3} \mathbf{C} P^{m+2} \rightarrow$ $S^{2 n-1}$. Notice that the composition $i \circ h$ is a self map of the sort studied in Theorem 3.4(ii).

It is straightforward to see that there is a localized version of Theorem 3.4 in which the numbers $F L_{n}$ are replaced by the highest power of $p$ which divide them. Since $i \circ h$ induces the vector $\left(p^{e}, 0, \ldots, 0\right)$ in the $p$-local version of $D(m+2, \mathbf{C})$, it follows that $p^{e}$ is at least as large as the $p$-primary factor of 1.c.m. $(1, \ldots, m+2)$.

This completes the proof for the case $k=2 n-4$ and $m<\infty$. The case $m=\infty$ follows at once. Moreover, since the order of the identity on $\Omega^{k} X$ is a nonincreasing function of $k$, the remaining cases of Theorem 4.5 are immediate consequences of the one just proved.

\section{REFERENCES}

1. J. F. Adams, Vector fields on spheres, Ann. of Math. (2) 75 (1962), 603-632.

2. __ On the groups $J(X)$. IV, Topology 5 (1966), 21-71.

3. __ Infinite loop spaces, Ann. of Math. Studies, no. 90, Princeton Univ. Press, Princeton, N.J., 1978.

4. M. Arkowitz and C. R. Curjel, On the maps of H-spaces, Topology 6 (1967), 137-148. 
5. M. F. Atiyah, Thom complexes, Proc. London Math. Soc. 11 (1961), 291-310.

6. J. C. Becker, Characteristic classes and K-theory, Lecture Notes in Math., vol. 428, Springer-Verlag, Berlin and New York, 1974, pp. 132-143.

7. F. R. Cohen, J. C. Moore and J. A. Neisendorfer, Torsion in homotopy, Ann. of Math. (2) 109 (1979), 121-168.

8. D. Davis, BP-operations and mappings of stunted complex projective spaces, Lecture Notes in Math., vol. 741, Springer-Verlag, Berlin and New York, 1979, pp. 373-393.

9. E. Dror, $A$ generalization of the Whitehead theorem, Lecture Notes in Math., vol. 249, Springer-Verlag, Berlin and New York, 1971, pp. 13-22.

10. E. Dyer, Chern characters of certain complexes, Math. Z. 80 (1963), 363-373.

11. S. Feder and S. Gitler, Mappings of quaternionic projective space, Bol. Soc. Mat. Mexicana 18 (1973), $33-37$.

12. P. Hilton, G. Mislin and J. Roitberg, Localization of nilpotent groups and spaces, North-Holland Math. Studies, no. 15, North-Holland, Amsterdam, 1976.

13. I. M. James, Cross-sections of Stiefel manifolds, Proc. London Math. Soc. 8 (1958), 536-547.

14. , The topologv of Stiefel manifolds, London Math. Soc. Lecture Notes Series, vol. 24, Cambridge Univ. Press, Cambridge, 1976.

15. H. J. Marcum and D. Randall, A note on self-mappings of quarternionic projective spaces, An. Acad. Brasil, Ciênc. 47 (1975), 7-9.

16. C. A. McGibbon, Multiplicative properties of power maps. II, Trans. Amer. Math. Soc. (to appear).

17. Stable properties of rank 1 loop structures, Topology 20 (1981), 109-118.

18. R. J. Milgram and E. Rees, On the normal bundle to an embedding, Topology 10 (1971), 299-308.

19. M. Mimura and H. Toda, On p-equivalences and p-universal spaces, Comment Math. Helv. 46 (1971), 87-97.

20. J. A. Neisendorfer, 3-primary exponents, Proc. Cambridge Philos. Soc. 90 (1981), 63-83.

21. J. A. Neisendorfer and P. Selick, Some examples of spaces with or without exponents, Proceedings of Current Trends in Algebraic Topology, London, Ontario, 1981 (to appear).

22. D. L. Rector, Loop structures on the homotopy type of $S^{3}$, Lecture Notes in Math., vol. 249, Springer-Verlag, Berlin and New York, 1971, pp. 99-105.

23. E. Rees, Embeddings of real projective spaces, Topology 10 (1971), 309-312.

24. J. P. Serre, $A$ course in arithmetic, Graduate Texts in Mathematics, no. 7, Springer-Verlag, Berlin and New York, 1970.

25. (j. Segal, The stable homotopy of complex projective space, Quart. J. Math. Oxford Ser. (2), 24 (1973), $1-5$.

26. P. Selick, Odd primary torsion in $\pi_{k}\left(S^{3}\right)$, Topology 17 (1978), 407-412.

27. V. P. Snaith, Algebraic cobordism and K-theory, Mem. Amer. Math. Soc., no. 221 (1979).

28. E. Spanier, Algebraic topologl, McGraw-Hill, New York, 1966.

29. N. E. Steenrod (with D. B. A. Epstein), Cohomology operations, Ann. of Math. Studies, no. 50, Princeton Univ. Press, Princeton, N.J., 1962.

30. D. Sullivan, Geometry topology: Part I, localization, periodicity, and Galois symmetry, MIT Notes, Cambridge. 1970.

31. H. Toda, Composition methods in homotopy groups of spheres, Ann. of Math. Studies, no. 49 , Princeton Univ. Press, Princeton, N.J., 1962.

32. A. Zabrodsky, p-equivalences and homotopy type, Lecture Notes in Math., vol. 418, Springer-Verlag, Berlin and New York, 1974, pp. 161-171.

33. Hopf spaces, North-Holland Math. Studies, no. 22, North-Holland, Amsterdam, 1976.

Department of Mathematics, Indiana University, Bloomington, INDIANa 47405 